\title{
Detecting Influenza Epidemics Using Self-reported Data Through Mobile App (FeverCoach)
}

\author{
Myeongchan Kim ${ }^{1,2}$, MD; Sehyo Yune ${ }^{2}$, MD, MPH, MBA; Hyun Wook $\mathrm{Han}^{3}, \mathrm{MD}, \mathrm{PhD}$ \\ ${ }^{1}$ MOBILE DOCTOR CO., LTD., Seoul, Republic Of Korea \\ ${ }^{2}$ MIDAS Research Center, Seoul, Republic Of Korea \\ ${ }^{3}$ Department of Biomedical Informatics, School of Medicine, Ajou University, Suwon, Republic Of Korea
}

\section{Corresponding Author:}

Hyun Wook Han, MD, PhD

Department of Biomedical Informatics

School of Medicine

Ajou University

206, World cup-ro

Suwon,

Republic Of Korea

Phone: 821062920812

Email: james.hw.han@gmail.com

\section{Abstract}

Background: Timely forecast of influenza activity is critical for a public health system to prepare for an influenza epidemic and mitigate its burden. Currently, influenza surveillance relies on traditional data sources such as reports from health care providers, which lag behind real-time by several days to weeks. In an effort to reduce the time lag, internet search information, voluntary web-based records, and electronic health records have been suggested as the alternative data sources for influenza surveillance. However, low specificity, low rate of report, or privacy concerns limits the use of such data.

Objective: FeverCoach mobile application provides tailored information to help caregivers manage a febrile child. Using the self-reported diagnosis data submitted to the app, we developed a new algorithm that accurately predicted the influenza trend in South Korea.

Methods: Users of FeverCoach agreed to the use of de-identified data for research purposes. The app shows information about use of antipyretics and adjuvant way to relieve fever when users enter the child's age, sex, body temperature, and the duration of fever. Users can choose from the list of 21 candidate diseases including Influenza after a physician office visit. Additional information about the disease was provided following submission of the diagnosis. Public influenza-like illness (ILI) data was obtained from the Korea Centers for Disease Control and Prevention (KCDC) website. The data was collected from September 2016 to March 2017. Ordinary least squares linear regression was used to build a model using the data from the app to predict the influenza trend. To perform linear regression, we calculate logit(Pcdc) and logit(Papp) where logit(p) is natural log of p/(1-p), Pcdc is (ILI visit counts)/(total patient visit counts) and Papp is (Influenza report on FeverCoach)/(total diagnosis report on FeverCoach).

Results: We collected 13,014 self-reported diagnoses. Of all users, $81 \%$ of the children were under 5 years of age. The animated visualization of spatiotemporal diagnosis report is available online at https://www.youtube.com/watch?v=-8kDXz43gO8. Ordinary least square regression showed significant association between logit(Pcdc) and logit(Papp) (R2=0.860, $P<.001)$. Using this regression model, we could detect an influenza epidemic 5 days before the 2016-2017 season's influenza epidemic alert by KCDC.

Conclusions: We found that it is possible to predict influenza epidemics earlier than KCDC with a relatively small amoount data. Collection of specific and accurate data was made possible by targeting a well-defined population.

(iproc 2017;3(1):e56) doi: 10.2196/iproc.8686

\section{KEYWORDS}

children; epidemics; health care; human influenza; Mobile health (mHealth) 
Figure 1. FeverCoach main screens. (a) input page for fever. (b) information for fever management. (c) input page for diagnosis.

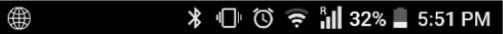

$<\quad$ Enter the temperature

What is the temperature of child?

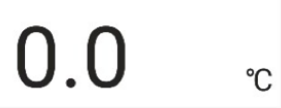

When did you measure the temperature?

2016-10-19 PM 05:51

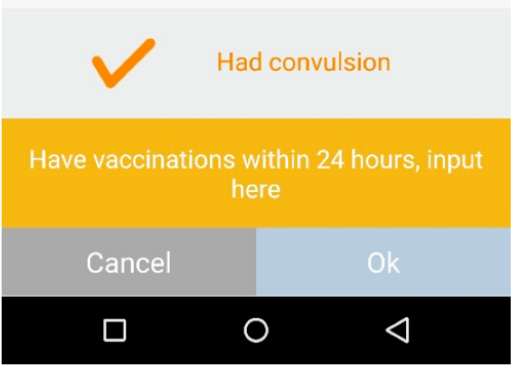

(a)

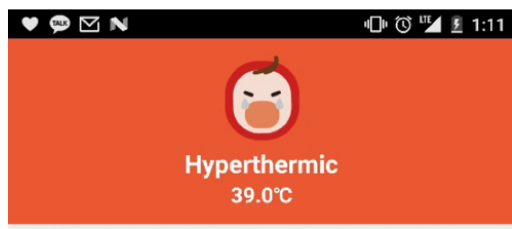

The child is hyperthermic. Measure again after 1 hours.

Consider giving antipyretics.

Use acetaminophen type or ibuprofen type. A proper dose is $5.0 \mathrm{cc} \sim 6.0 \mathrm{cc}$.

Antipyretics $\mathbf{\nabla}$

Make sure to give the child a plenty of water to avoid dehydration.

Calculate the water intake

Wipe off face, neck, chest and armpits of the child with lukewarm water if the child is the child with lukewarm

If the child is given antipyretic, please wait

30 minutes before wiping the child's body.

Instructions for lukewarm water massage $\mathbf{V}$

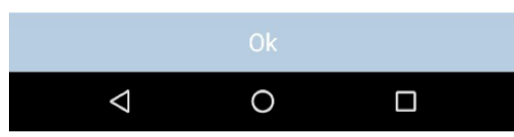

(b)

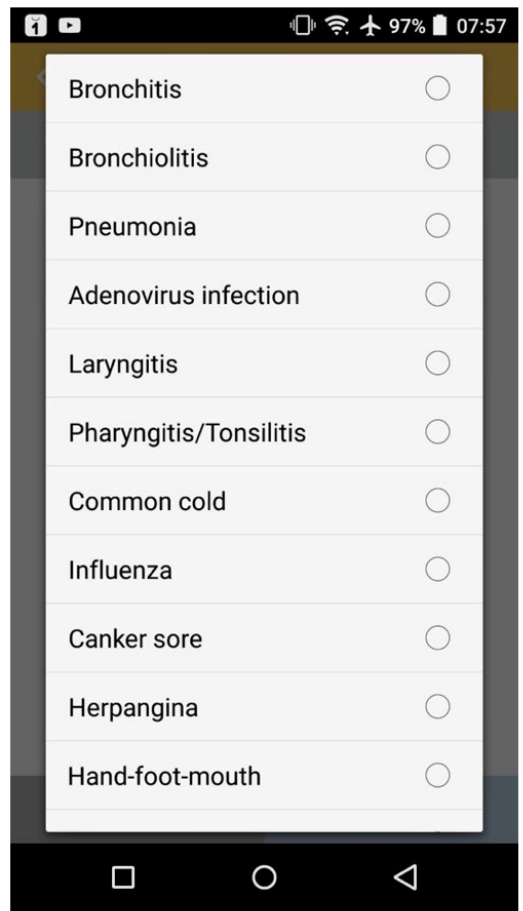

(c)

Figure 2. Visualization of spatiotemporal diagnosis report. Green circles indicate diagnosis data except Influenza and Red circles show input of Influenza diagnosis. Diameters of circles present time proximity from each reporting date of diagnosis. (a) Influenza reports on the 25th November, 2016. (b) Influenza reports on December 7th, 2016 : a day before epidemic alert of KCDC. (c) status on December 23rd, 2016. (d) status on January 28 th, 2017.

(a)

$11-25$

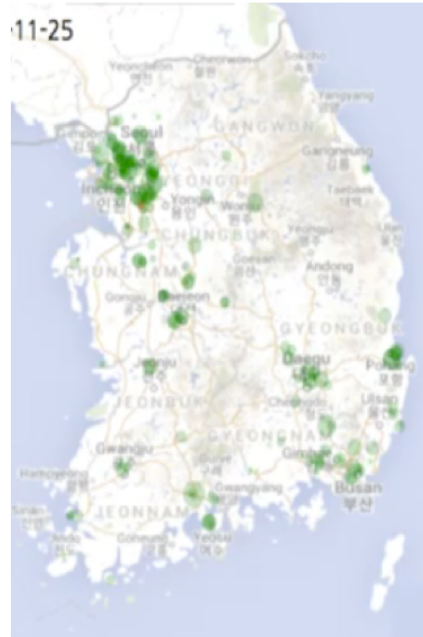

(b)

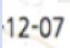

(d)

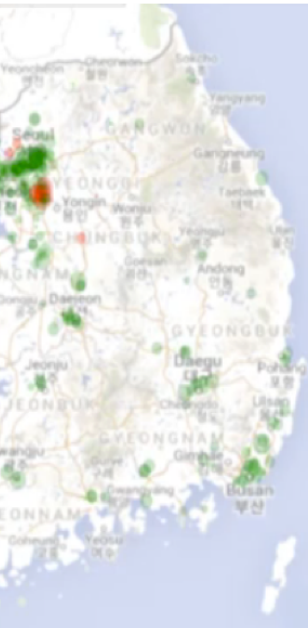

(c)

\section{$12-23$}

(c)

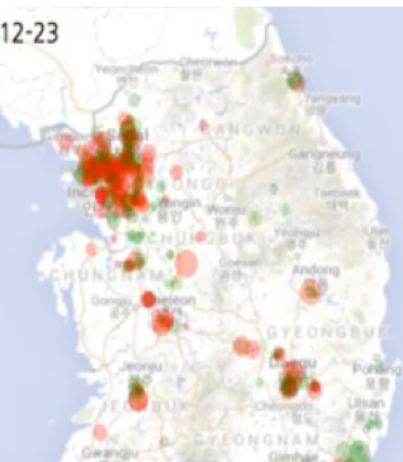

$01-28$

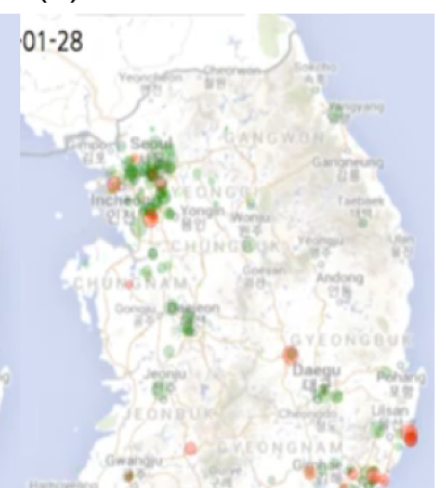


Figure 3. A comparison between CDC ILI data and FeverCoach data arranged by detection date : Pcdc $=($ ILI visit counts)/(total patient visit counts) and Papp $=($ Influenza report on FeverCoach $) /($ total diagnosis report on FeverCoach $)$. Red line shows prediction of our regression model , blue line shows logit (Pcdc) and green line shows KCDC's influenza epidemic alert standard : fraction of ILI visit $=0.0089$.

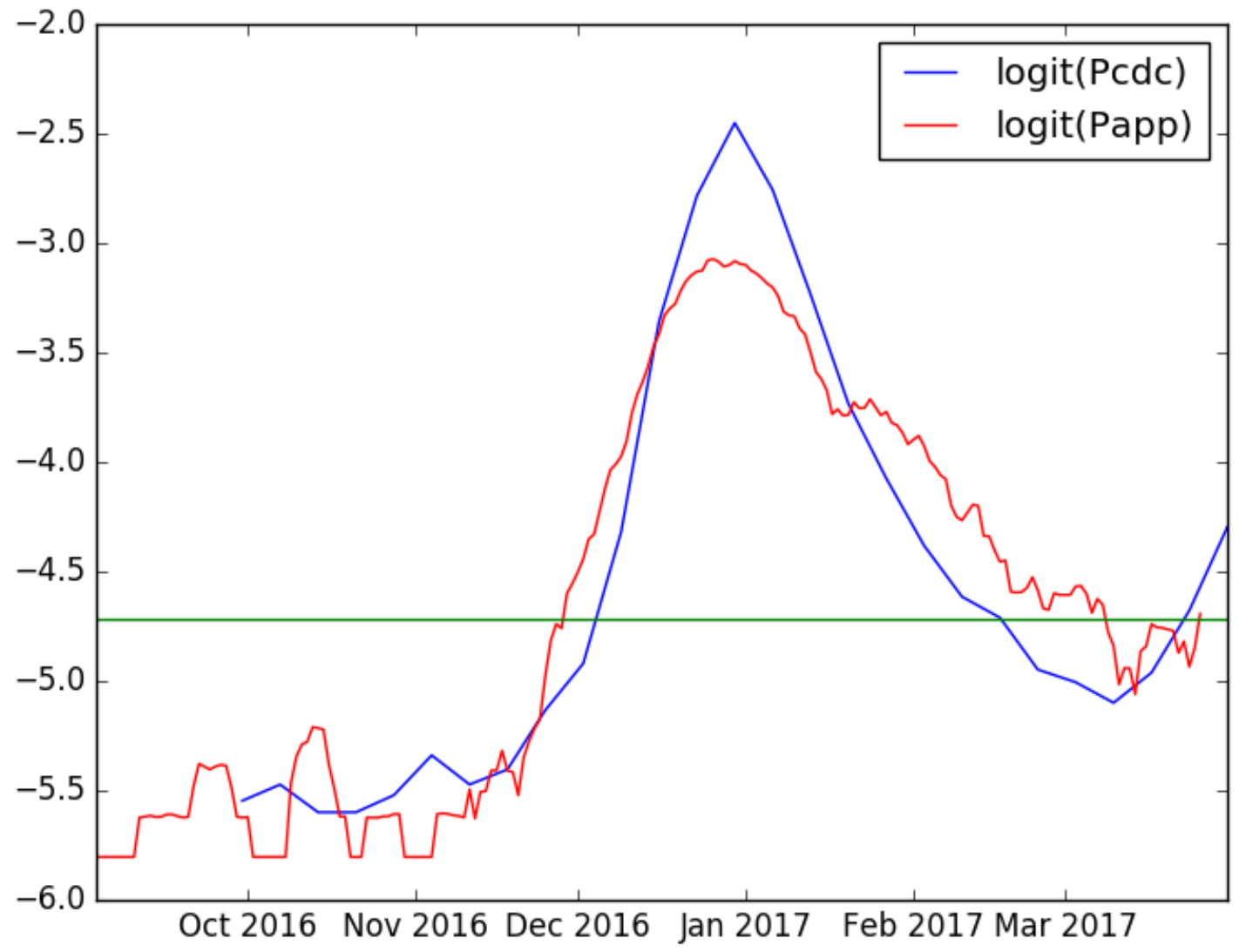

\section{Multimedia Appendix 1}

Data visualization of influenza report through FeverCoach from 2016 Nov to 2017 Jan. Red circles mean influenza report, green circles mean other diagnosis report. Circles are appeared from 5 days before of reporting date, and they have the biggest size on reporting date. Shrinking and disappearing takes 5 days.

[MOV File, 8MB-Multimedia Appendix 1]

\section{Multimedia Appendix 2}

Full poster.

[PDF File (Adobe PDF File), 9MB-Multimedia Appendix 2]

Edited by T Hale; this is a non-peer-reviewed article. Submitted 10.08.17; accepted 25.08.17; published 22.09.17.

Please cite as:

Kim M, Yune S, Han HW

Detecting Influenza Epidemics Using Self-reported Data Through Mobile App (FeverCoach)

iproc 2017;3(1):e56

URL: http://www.iproc.org/2017/1/e56/

doi: $\underline{10.2196 / \text { iproc. } 8686}$

PMID: 
CMyeongchan Kim, Sehyo Yune, Hyun Wook Han. Originally published in Iproceedings (http://www.iproc.org), 22.09.2017. This is an open-access article distributed under the terms of the Creative Commons Attribution License (https://creativecommons.org/licenses/by/4.0/), which permits unrestricted use, distribution, and reproduction in any medium, provided the original work, first published in Iproceedings, is properly cited. The complete bibliographic information, a link to the original publication on http://www.iproc.org/, as well as this copyright and license information must be included. 